\title{
VARIABILIDADE ESPACIAL DE ATRIBUTOS DO SOLO ANTES E APÓS CALAGEM E FOSFATAGEM EM DOSES VARIADAS NA CULTURA DE CANA-DE-AÇÚCAR ${ }^{1}$
}

\author{
JOSÉ E. CORÁ ${ }^{2}$, JOSÉ M. G. BERALDO ${ }^{3}$
}

\begin{abstract}
RESUMO: Avaliou-se a distribuição espacial de atributos do solo por meio de mapas de isolinhas elaborados a partir da variabilidade espacial dos atributos expressa no semivariograma e por meio de interpolação linear, antes e após a aplicação de doses variadas de calcário e fertilizante fosfatado na cultura de cana-de-açúcar. Foram coletadas amostras de solo na intensidade de uma amostra por hectare, na profundidade de 0-0,20 m, em três talhões comerciais de cana-de-açúcar. Os mapas de isolinhas elaborados por meio da krigagem, levando-se em consideração a existência de dependência espacial de atributos do solo, expressa no semivariograma, apresentaram maior precisão e diferentes padrões de ocorrência da distribuição espacial dos atributos, comparados àqueles construídos utilizando-se do interpolador linear para a estimativa dos valores em locais não medidos. A densidade de amostragem igual a uma amostra por ha não foi suficiente para revelar a dependência espacial dos teores de $\mathrm{P}$ e valores de $\mathrm{V}$ no talhão $\mathrm{C}$, e de $\mathrm{V}$ no talhão $\mathrm{A}$. Os talhões $\mathrm{A}$ e B apresentaram maior continuidade espacial para os teores de $\mathrm{P}$ e o talhão B maior continuidade espacial para V. Houve aumento da continuidade espacial dos atributos depois de realizada a calagem e a fosfatagem em doses variadas.
\end{abstract}

PALAVRAS-CHAVE: agricultura de precisão, manejo do solo, geoestatística.

\section{SPATIAL VARIABILITY OF SOIL PROPERTIES BEFORE AND AFTER LIME AND PHOSPHORUS FERTILIZER APPLICATION AT VARIABLE RATES IN SUGARCANE}

ABSTRACT: The objective of this work was to evaluate the spatial variability of soil properties by soil maps developed by different methods, before and after liming and phosphorus fertilizer application at variable rates. Soil georeferenced samples were obtained from $0-0,20 \mathrm{~m}$ depths in three sugarcane fields (A, B and C). Lime and phosphorus requirement were calculated and applied at variable rates. Soil maps generated from semivariogram parameters showed different patterns and were more precise than those generated from linear interpolation method. Liming and phosphorus variable rate application reduced the spatial variability of base saturation (V) and phosphorus level (P) in the experimental area. The soil sampling intensity equal to one per ha used in this work was not sufficient to assess the spatial variability of $\mathrm{P}$ and $\mathrm{V}$ levels in the $\mathrm{C}$ field and the $\mathrm{V}$ levels in the B field.

KEYWORDS: precision agriculture, site-specific-management, geostatistics.

\section{INTRODUÇÃO}

A agricultura de precisão inicia-se pelo reconhecimento da variabilidade espacial da produtividade das culturas em uma determinada área. A segunda etapa consiste na determinação e na investigação dos fatores de produção responsáveis por essa variabilidade. Uma terceira etapa consiste em utilizar-se desses conhecimentos para ajustar um manejo específico na área, proporcionando a aplicação de insumos e/ou práticas agrícolas, de acordo com a variabilidade encontrada nos fatores de produção investigados, com o objetivo de evitar excessos ou faltas na aplicação dos mesmos, o que poderia afetar o desenvolvimento das culturas. A etapa seguinte seria

\footnotetext{
${ }^{1}$ Extraído da dissertação de Mestrado do segundo autor.

${ }^{2}$ Prof. Doutor, Departamento de Solos e Adubos, UNESP - Jaboticabal - SP, Fone: (0XX16) 3209.2672, cora@ fcav.unesp.br

${ }^{3}$ Eng ${ }^{\circ}$ Agrônomo, Mestre em Produção Vegetal, IAPAR, Paranavaí - PR, jmgberaldo@iapar.br

Recebido pelo Conselho Editorial em: 3-12-2004

Aprovado pelo Conselho Editorial em: 24/5/2006
} 
a avaliação da eficiência do manejo específico adotado por meio da avaliação do comportamento da produtividade da cultura, que, por sua vez, é a primeira etapa de um novo ciclo.

Entre os fatores de produção responsáveis pela variabilidade espacial da produtividade das culturas, os atributos do solo assumem grande importância, pois, em muitos casos, não variam no espaço e no tempo ao acaso, mas de acordo com uma continuidade aparente ou dependência espacial. A dependência espacial pode ser estimada pelo semivariograma que, por sua vez, pode ser obtido por meio de análise geoestatística dos dados (TRANGMAR et al., 1985).

Uma das maneiras mais utilizadas para representar a variabilidade espacial dos atributos do solo em uma área é por meio de mapas de isolinhas (BURGESS \& WEBSTER, 1980). Dentro do conceito de agricultura de precisão, são definidos três tipos de mapas de isolinhas: mapas de condição, mapas de recomendação ou prescrição e mapas de desempenho. Os mapas de condição mostram a distribuição espacial dos parâmetros avaliados em uma determinada área, antes de qualquer intervenção. Mapas de recomendação são derivados de um ou mais mapas de condição e contêm informações sobre as quantidades necessárias de insumos e/ou práticas agrícolas que cada local específico da área deverá receber (manejo específico), respeitando a variabilidade dos atributos avaliados (mapas de condição), sendo a base para a tecnologia da aplicação em doses variáveis. O mapa de desempenho permite avaliar como a área se apresenta após o manejo específico, caracterizando-se também como mapa de condição para um próximo ciclo no contexto da agricultura de precisão.

É necessário que os mapas de recomendação sejam de alta precisão para que seja possível a aplicação do manejo específico, adequadamente. A precisão dos mapas depende de fatores, como intensidade e esquema da amostragem e da escolha do método de interpolação dos dados, por influenciarem na estimativa dos valores dos atributos do solo em locais não amostrados.

Os métodos de interpolação mais usados no mapeamento dos atributos do solo são aqueles que utilizam interpolação linear ou polinômios e o método da krigagem (SCHLOEDER et al., 2001). Ambos estimam valores em locais não amostrados dentro da área de estudo. Contudo, a forma na qual os dados variam de um local para outro no campo, não necessariamente, segue equações lineares ou polinômios. Quando isso acontece, muitas vezes, na prática, a forma e o grau do polinômio não suportam nenhuma interpretação física para o fenômeno, fato que é revelado no semivariograma (VIEIRA, 2000). Em contrapartida, obtendo-se o semivariograma da variável e constatada a dependência espacial entre as amostras, a krigagem possibilita a estimativa dos valores em qualquer posição dentro do campo de estudo, sem tendência e com variância mínima, duas características que fazem dela um interpolador ótimo (BURGESS \& WEBSTER, 1980). Desse fato, resulta maior exatidão na estimativa dos valores das variáveis em locais não amostrados e, conseqüentemente, proporciona maior precisão na confecção dos mapas.

Entretanto, tem-se verificado que consultores agronômicos, durante o processo de elaboração dos mapas de isolinhas, utilizando programas de computador disponíveis no mercado para suporte em agricultura de precisão, não levam em consideração a dependência espacial dos atributos analisados para estimar valores em locais não-amostrados. Eles elaboram os mapas utilizando métodos baseados em interpolação linear ou polinômios, deixando dúvida em relação à acurácia dos mapas. A razão para tal fato é que tais profissionais são simples usuários dos programas e, na maioria das vezes, não têm conhecimentos científicos necessários sobre o que está envolvido no processo de elaboração dos mapas pelos programas, não questionando, portanto, a precisão dos mesmos.

O objetivo deste trabalho foi avaliar a distribuição espacial de atributos do solo por meio de mapas de isolinhas elaborados partindo-se da variabilidade espacial de atributos do solo expressa no semivariograma e por meio de interpolação linear, antes e após a aplicação de calcário e fertilizante fosfatado em doses variadas. 


\section{MATERIAL E MÉTODOS}

A área experimental localiza-se no município de Guariba - SP, cujas coordenadas estão situadas a $21^{\circ} 24^{\prime}$ de latitude sul e $48^{\circ} 07^{\prime}$ de longitude oeste, com altitude média de $530 \mathrm{~m}$, predominando o relevo suave ondulado, sendo selecionados três talhões de cana-de-açúcar sob reforma. O mapeamento dos solos da área, realizado na escala de 1:5.000 (PRADO, 1994), revelou que os talhões A, com 25,8 ha, e B, com 19,0 ha, se encontram sob Latossolo Vermelho distroférrico textura argilosa, e o talhão C, medindo 24,4 ha, parte se encontra sob Latossolo Vermelho eutroférrico textura argilosa e parte sob Latossolo Vermelho eutrófico textura média.

Em agosto de 2002, após a colheita da cana-de-açúcar, foram realizadas amostragens de solo nos três talhões, sendo em grade regular e na intensidade de uma amostra por hectare, na profundidade de $0-0,20 \mathrm{~m}$. A intensidade de amostragem igual a uma amostra por hectare foi definida baseando-se no que é comumente recomendado por consultores e que já vinha sendo realizado pelo proprietário da área. Ela teve como objetivo testar se o realizado pelo proprietário (uma amostra por ha) era suficiente para ter boa estimativa da variabilidade espacial dos atributos do solo e se estava adequado para elaborar os mapas de isolinhas com a precisão adequada. Portanto, foram coletadas 26 amostras no talhão A (25,8 ha), 20 amostras no talhão B (19,0 ha) e 23 amostras no talhão C (24,4 ha). Em cada ponto de amostragem, foram coletadas, com trado tipo holandês, dez subamostras de material de solo, aleatoriamente, num raio de $10 \mathrm{~m}$ do ponto central, para compor uma amostra composta representativa do ponto de amostragem. Os pontos de amostragem e o perímetro de cada talhão foram georreferenciados utilizando-se de sistema de posicionamento global de navegação (GPS).

As amostras de solo foram preparadas e submetidas às análises químicas para fins de fertilidade. Nas amostras, foram determinados os teores de $\mathrm{P}, \mathrm{Ca}, \mathrm{Mg}, \mathrm{K}$ e $\mathrm{H}+\mathrm{Al}$ e calculadas a capacidade de troca de cátions do solo (CTC) e a saturação por bases do solo (V). Com esses dados, calcularam-se a necessidade de calagem (NC) e a necessidade de fosfatagem (NF) do solo para cada amostra composta. A NC foi calculada para elevar a saturação por bases do solo a 70\%, utilizando-se do calcário dolomítico com PRNT de 70\% e a NF para elevar os teores de fósforo no solo para $40 \mathrm{mg} \mathrm{dm}^{-3}$. A fonte de fósforo utilizada foi o fosfato reativo Daoui na forma farelada.

A distribuição do calcário e do fosfato foi realizada em doses variadas, tendo como base os mapas de recomendação da NC e da NF, confeccionados previamente a partir dos mapas de condição inicial dos atributos do solo. Utilizou-se, para confecção dos mapas, do programa SSToolbox v.3.4 (SST Development Group). A aplicação dos insumos foi realizada em 15-8-2002, utilizando-se de distribuidora marca Nevoeiro, modelo 10.006, para produtos granulados ou em pó. Após a aplicação, o calcário e o fosfato foram incorporados ao solo por meio de gradagem realizada na profundidade de $0,20 \mathrm{~m}$, com posterior nivelamento da superfície do solo com grade niveladora. Procedeu-se à semeadura da soja na primeira quinzena de novembro de 2002, cuja adubação de plantio constituiu-se de $220 \mathrm{~kg} \mathrm{ha}^{-1}$ da fórmula 01-32-00, distribuídos na linha de semeadura.

Após a colheita da soja, em março de 2003, foram coletadas amostras de solo nos mesmos pontos da amostragem inicial, com o objetivo de obter os dados para a confecção dos mapas de isolinhas dos atributos dos solos avaliados após o manejo específico (mapas de desempenho). Os dados foram submetidos às análises estatísticas descritivas, e a hipótese de normalidade foi testada pelo teste W a 5\% (SHAPIRO \& WILK, 1965). Os dados que não apresentaram distribuição normal foram transformados em sua forma logarítmica $(\mathrm{y}=\ln \mathrm{x})$ e novamente submetidos ao teste W. Após a transformação, os dados apresentaram distribuição normal. A análise de dependência espacial das variáveis foi efetuada pelo ajuste dos dados ao semivariograma experimental (análise geoestatística), de acordo com a teoria das variáveis regionalizadas (TVR), utilizando-se do programa GS+ (ROBERTSON, 1998).

Na seleção dos modelos dos semivariogramas, foram considerados os valores de $R^{2}$ e $S Q R$. Segundo ROBERTSON (1998), a SQR é um parâmetro mais robusto do que o $\mathrm{R}^{2}$ e propicia 
medida exata de quão bem o modelo se ajusta aos dados. O programa GS+ utiliza a combinação de parâmetros do semivariograma para minimizar a SQR para cada modelo. Os modelos dos semivariogramas ajustados foram validados pela técnica de validação cruzada (CRESSIE, 1991). Conhecidos os semivariogramas das variáveis e sendo observada a dependência espacial entre as amostras, os mapas de isolinhas de distribuição espacial das variáveis foram gerados pelo programa Surfer 6.01 (GOLDEN SOFTWARE, 1995).

Para estimar valores para qualquer local onde não se teve valor medido, utilizou-se a krigagem ordinária, levando-se em conta os parâmetros do semivariograma (modelo ajustado, efeito pepita, alcance e patamar) determinados pela análise geoestatística. Quando os atributos apresentaram ausência de dependência espacial (efeito pepita), utilizou-se da interpolação linear baseada no inverso do quadrado das distâncias para estimar os valores das variáveis em locais não amostrados. Esses mapas foram comparados visualmente com aqueles gerados pelo programa SSToolbox, o qual, no cálculo da estimativa dos valores das variáveis para locais não amostrados (interpolação), utiliza combinação linear dos valores medidos, ou seja, uma somatória dos valores medidos envolvidos na estimativa $(\mathrm{N})$, multiplicados pelos pesos associados a cada valor medido. Por exemplo, na interpolação linear, os pesos são todos iguais a 1/N e, na interpolação baseada no inverso do quadrado das distâncias, os pesos recebem valores variáveis de acordo com o inverso do quadrado das distâncias que separa o valor interpolado dos valores medidos. Os resultados são bastante semelhantes, utilizando-se de uma ou de outra combinação linear.

\section{RESULTADOS E DISCUSSÃO}

De maneira geral, os valores da média e da mediana para os atributos estudados foram similares, com as medianas apresentando valores pouco menores que a média na maioria dos casos, apesar da ocorrência de algumas distribuições assimétricas (Tabelas 1; 2 e 3). Segundo CAMBARDELLA et al. (1994), isso pode ser indicativo de que as medidas de tendência central não são dominadas por valores atípicos na distribuição. De qualquer forma, e de acordo com CRESSIE (1991), a normalidade dos dados não é uma exigência da geoestatística.

TABELA 1. Resultados da análise estatística descritiva para os teores de $\mathrm{P}\left(\mathrm{mg} \mathrm{dm}^{-3}\right)$, nos talhões A, B e C, nas condições de antes e depois da aplicação do fertilizante fosfatado em doses variadas.

\begin{tabular}{|c|c|c|c|c|c|c|c|c|}
\hline \multirow{2}{*}{ Condição } & \multirow{2}{*}{ Média } & \multirow{2}{*}{ Mediana } & \multicolumn{2}{|c|}{ Valores } & \multirow{2}{*}{$\begin{array}{l}\text { Desvio- } \\
\text { Padrão }\end{array}$} & \multicolumn{3}{|c|}{ Coeficientes $^{(1)}$} \\
\hline & & & Mínimo & Máximo & & Variação (\%) & Curtose & Assimetria \\
\hline \multicolumn{9}{|c|}{ Talhão A } \\
\hline Antes & 23 & 23 & 9 & 50 & 9,2 & 39 & 1,5 & 0,9 \\
\hline Após & 40 & 39 & 19 & 88 & 15,3 & 38 & 3,0 & 1,5 \\
\hline \multicolumn{9}{|c|}{ Talhão B } \\
\hline Antes & 15 & 14 & 9 & 31 & 50,2 & 46 & 0,3 & 1,2 \\
\hline Após & 49 & 43 & 21 & 85 & 18,3 & 38 & $-0,7$ & 0,5 \\
\hline \multicolumn{9}{|c|}{ Talhão C } \\
\hline Antes & 12 & 11 & 9 & 21 & 3,6 & 29 & 0,0 & 1,0 \\
\hline Após & 33 & 28 & 20 & 77 & 12,6 & 39 & 6,4 & 2,2 \\
\hline
\end{tabular}

${ }^{(1)}$ Os dados de coeficientes de variação, assimetria e curtose são adimensionais.

Observa-se, na Tabela 1, que, nos talhões A e B, os teores médios de $\mathrm{P}$ no solo atingiram o nível esperado após a adubação fosfatada, ou seja, $40 \mathrm{mg} \mathrm{dm}^{-3}$. $\mathrm{O}$ mesmo não foi observado no talhão $\mathrm{C}$, provavelmente, devido à diferente reatividade do fertilizante com os diferentes solos de ocorrência nesse talhão.

Houve aumento na amplitude dos teores de P no solo após a aplicação do fosfato em doses variadas, nos três talhões estudados (Tabela 1), devido ao aumento dos valores de máximo. Observou-se aumento dos valores de desvio-padrão para os talhões A e C e diminuição para o 
talhão B. Contudo, foram observados menores valores de coeficientes de variação (C.V.) após a aplicação de fosfatado em doses variadas, exceto para os teores de $\mathrm{P}$ do talhão $\mathrm{C}$. O maior valor de C.V. observado no talhão $\mathrm{C}$, provavelmente, também pode ser explicado pelo fato de que, nesse talhão, ocorrem dois solos diferentes, o que proporciona comportamento diferenciado do insumo e, conseqüentemente, maior variação dos teores de $\mathrm{P}$ na área.

Para os três talhões estudados, os valores médios de $\mathrm{V}$ não atingiram o esperado com a calagem, ou seja, $\mathrm{V}=70 \%$, entretanto ficaram próximos de $60 \%$ (Tabela 2 ). Trabalhos na literatura (NATALE \& COUTINHO, 1994) demonstram que, para atender à legislação brasileira vigente (Instrução Normativa do Ministério da Agricultura - IN no 4 de 2-4-2004), os valores do fator reatividade (RE), utilizados no cálculo do PRNT do calcário, estão superestimados, o que induz a um valor também superestimado do PRNT, que, por sua vez, induz ao cálculo de menor quantidade de calcário a ser aplicada, insuficiente para atingir os valores de V desejados. Portanto, esse fato, provavelmente, explica os resultados obtidos no presente estudo.

Diferentemente dos resultados obtidos para o teores de $\mathrm{P}$ no solo, não foi observado aumento da amplitude para os valores de V, após a aplicação de calcário em doses variadas. Nesse caso, os valores se mantiveram bem próximos para os três talhões. Observou-se diminuição do valor do desvio-padrão para V somente para o talhão C. Contudo, houve redução dos valores de C.V. após a calagem, em doses variadas, sendo a maior redução observada no talhão B. Maiores valores de C.V. foram encontrados para os teores de P no solo comparados aos valores de C.V. para V. Esse fato indica maior variabilidade de P no solo comparado com V. SOUZA et al. (1998) obtiveram valores de C.V. extremamente altos para os teores de $\mathrm{P}$ no solo. Os autores atribuíram a alta variabilidade aos efeitos da aplicação contínua de fertilizantes na linha de plantio, o que proporciona a existência de locais com valores extremamente altos ou baixos. Também os autores atribuíram a alta variabilidade a erros na amostragem. Provavelmente, a adubação com P, realizada na linha por ocasião da semeadura da soja, pode ter influenciado no aumento dos valores de C.V. para os teores de $\mathrm{P}$ no solo no presente estudo.

Devido à variação dos teores de $\mathrm{P}$ no solo, e valores de $\mathrm{V}$ nos diferentes talhões na condição inicial, as quantidades de calcário e de fosfato recomendadas para aplicação variaram consideravelmente. Nos talhões A e C, observam-se locais onde não foi necessária a aplicação de calcário e, em outros, a recomendação foi de até 2,3 e 2,8 t ha ${ }^{-1}$, respectivamente. Da mesma maneira, no talhão $\mathrm{B}$, houve locais em que a quantidade de calcário recomendada foi de até quatro vezes maior que em outros (Tabela 3).

TABELA 2. Resultados da análise estatística descritiva para os valores de V, nos talhões A, B e C, na condição de antes e depois da aplicação de calcário em doses variadas.

\begin{tabular}{|c|c|c|c|c|c|c|c|c|}
\hline \multirow{2}{*}{ Condiçãa } & \multirow{2}{*}{ Média } & \multirow{2}{*}{ Mediana } & \multicolumn{2}{|c|}{ Valores } & \multirow{2}{*}{$\begin{array}{l}\text { Desvio- } \\
\text { Padrão }\end{array}$} & \multicolumn{3}{|c|}{ Coeficientes $^{(1)}$} \\
\hline & & & Mínimo & Máximo & & Variação (\%) & Curtose & Assimetria \\
\hline \multicolumn{9}{|c|}{ Talhão A } \\
\hline Antes & 48 & 47 & 31 & 74 & 8,8 & 18 & 2,2 & 1,1 \\
\hline Após & 60 & 58 & 48 & 85 & 8,4 & 14 & 1,9 & 1,2 \\
\hline \multicolumn{9}{|c|}{ Talhão B } \\
\hline Antes & 33 & 31 & 18 & 56 & 10,6 & 33 & 0,0 & 0,7 \\
\hline Após & 57 & 58 & 34 & 73 & 10,3 & 18 & $-0,5$ & $-0,4$ \\
\hline \multicolumn{9}{|c|}{ Talhão C } \\
\hline Antes & 51 & 52 & 28 & 73 & 12,7 & 25 & $-0,7$ & $-0,3$ \\
\hline Após & 63 & 61 & 45 & 86 & 8,9 & 14 & 0,9 & 0,6 \\
\hline
\end{tabular}


TABELA 3. Resultados da análise estatística descritiva para os valores da $\mathrm{NC}$ e $\mathrm{NF}\left(\mathrm{t} \mathrm{ha}^{-1}\right)$ nos talhões A, B e C.

\begin{tabular}{|c|c|c|c|c|c|c|c|c|}
\hline \multirow{2}{*}{ Atributo } & \multirow{2}{*}{ Média } & \multirow{2}{*}{ Mediana } & \multicolumn{2}{|c|}{ Valores } & \multirow{2}{*}{$\begin{array}{l}\text { Desvio- } \\
\text { Padrão }\end{array}$} & \multicolumn{3}{|c|}{ Coeficientes $^{(1)}$} \\
\hline & & & Mínimo & Máximo & & Variação (\%) & Curtose & Assimetria \\
\hline \multicolumn{9}{|c|}{ Talhão A } \\
\hline $\mathrm{NC}$ & 1,4 & 1,5 & 0,0 & 2,3 & 0,5 & 37 & 1,2 & $-1,0$ \\
\hline $\mathrm{NF}$ & 0,6 & 0,6 & 0,4 & 0,8 & 0,1 & 17 & $-0,1$ & $-0,2$ \\
\hline \multicolumn{9}{|c|}{ Talhão B } \\
\hline $\mathrm{NC}$ & 2,5 & 2,4 & 1,0 & 4,0 & 0,8 & 32 & $-0,4$ & 0,7 \\
\hline $\mathrm{NF}$ & 0,7 & 0,7 & 0,5 & 0,8 & 0,1 & 14 & 0,2 & $-1,1$ \\
\hline \multicolumn{9}{|c|}{ Talhão C } \\
\hline $\mathrm{NC}$ & 1,3 & 1,2 & 0,0 & 2,8 & 0,9 & 66 & $-1,1$ & 0,2 \\
\hline $\mathrm{NF}$ & 0,7 & 0,8 & 0,6 & 0,8 & 0 & 7 & 0,0 & $-1,0$ \\
\hline
\end{tabular}

As amplitudes da necessidade de fosfatagem foram menores para os diferentes talhões, quando comparadas àquelas referentes à necessidade de calagem (Tabela 3). Mesmo assim, podese observar, no talhão $\mathrm{A}$, que existe local onde a recomendação foi o dobro que em outros. Observaram-se, ainda, menores valores de desvio-padrão e C.V. para os dados de necessidade de fosfatagem quando comparados àqueles da necessidade de calagem.

As quantidades totais de calcário e de fosfato calculadas, considerando-se os valores médios ou os valores específicos de cada local, foram semelhantes (Tabela 4). Contudo, na aplicação em doses variadas, os insumos foram alocados nos locais e nas quantidades adequadas, respeitando a variabilidade espacial dos atributos dos solos, evitando, dessa maneira, excesso ou falta em determinados locais, possibilitando maior otimização do mesmo.

TABELA 4. Quantidades totais de calcário e fosfato $\left(\mathrm{t} \mathrm{ha}^{-1}\right)$ calculadas, considerando os valores médios de $\mathrm{V}$ e $\mathrm{P}$ e os específicos para cada local de amostragem nos talhões $\mathrm{A}, \mathrm{B}$ e C.

\begin{tabular}{cccccc}
\hline \multirow{2}{*}{ Talhão } & \multicolumn{3}{c}{ NC } & & \multicolumn{2}{c}{ NF } \\
\cline { 2 - 3 } \cline { 5 - 6 } \cline { 5 - 6 } & Média & Aplicada & & Média & Aplicada \\
A & 36,1 & 29,7 & & 15,4 & 15,2 \\
B & 47,5 & 47,6 & & 13,3 & 12,9 \\
C & 31,7 & 32,6 & & 17,0 & 17,9 \\
\hline
\end{tabular}

Os teores de $\mathrm{P}$ no solo apresentaram dependência espacial antes e após a aplicação do fertilizante fosfatado em doses variadas, nos talhões A e B (Figuras 1a, 1b, 1c e 1d), cujos dados foram ajustados ao modelo esférico. No talhão C, observou-se efeito pepita puro ou ausência total de dependência espacial para os teores de P no solo, ou seja, a distribuição foi aleatória, tanto antes como depois de realizada a fosfatagem (Figura 1e e 1f).

$\mathrm{O}$ valor do alcance de dependência espacial para os teores de $\mathrm{P}$ do solo foi maior no talhão $\mathrm{A}$, comparado àquele do talhão $\mathrm{B}$, tanto antes como depois de realizada a fosfatagem. Em ambos os talhões, o teor de $\mathrm{P}$ no solo apresentou maior valor do alcance depois de realizada a fosfatagem em doses variadas, indicando maior continuidade espacial dos teores de $\mathrm{P}$ no solo após a aplicação do insumo, respeitando a variabilidade espacial intrínseca dos atributos solo.

Os valores de $\mathrm{V}$ apresentaram dependência espacial somente no talhão $\mathrm{B}$, tanto antes como depois de realizada a calagem em doses variadas (Figura 2), cujos dados foram ajustados ao modelo esférico. Da mesma maneira que ocorreu para os teores de $\mathrm{P}$ no solo, o maior valor de alcance para $\mathrm{V}$ foi observado depois de realizada a calagem em doses variadas. 

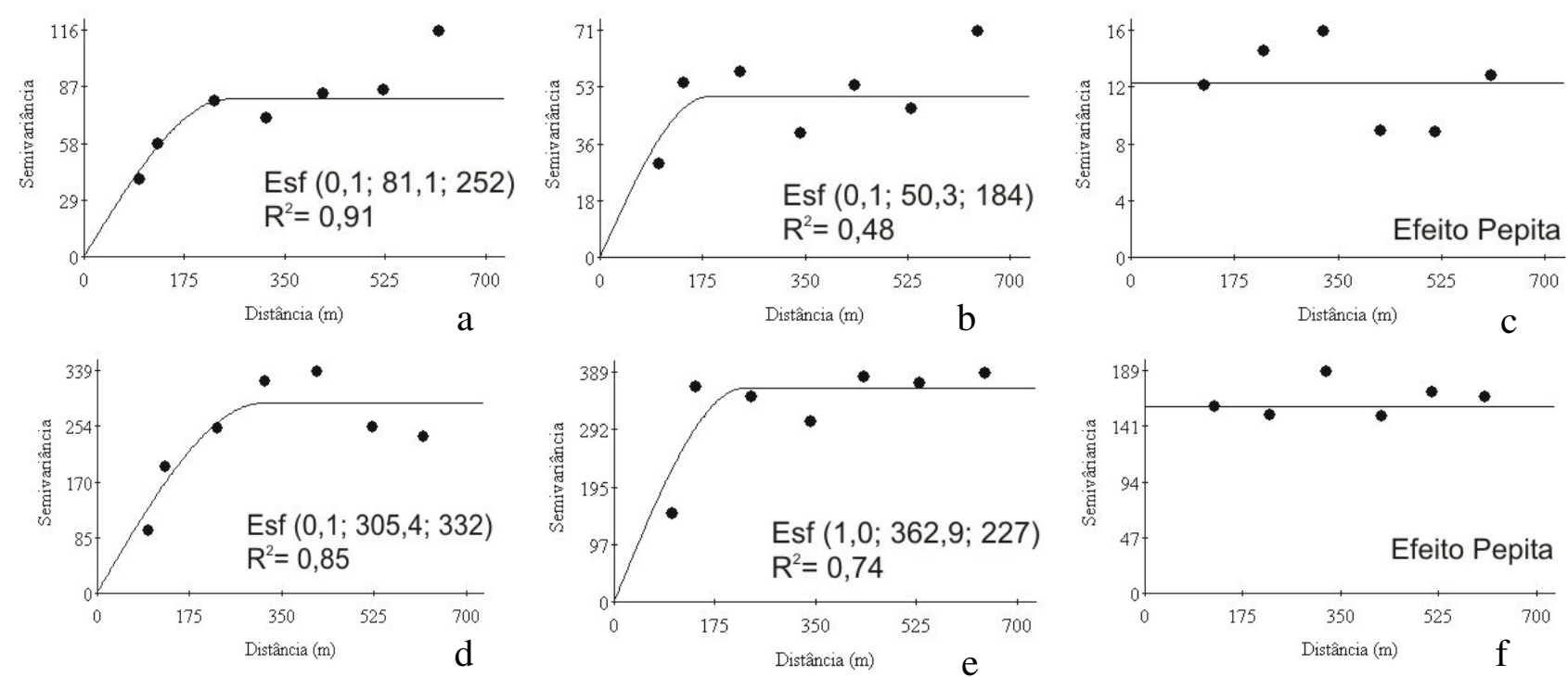

FIGURA 1. Semivariogramas ajustados para os teores de $\mathrm{P}\left(\mathrm{mg} \mathrm{dm}^{-3}\right)$ do solo na condição de antes (a, c, e) e após (b, d, f) a aplicação em doses variadas, nos talhões A, B e C, respectivamente. Valores entre parênteses são, respectivamente, efeito pepita, patamar e alcance.
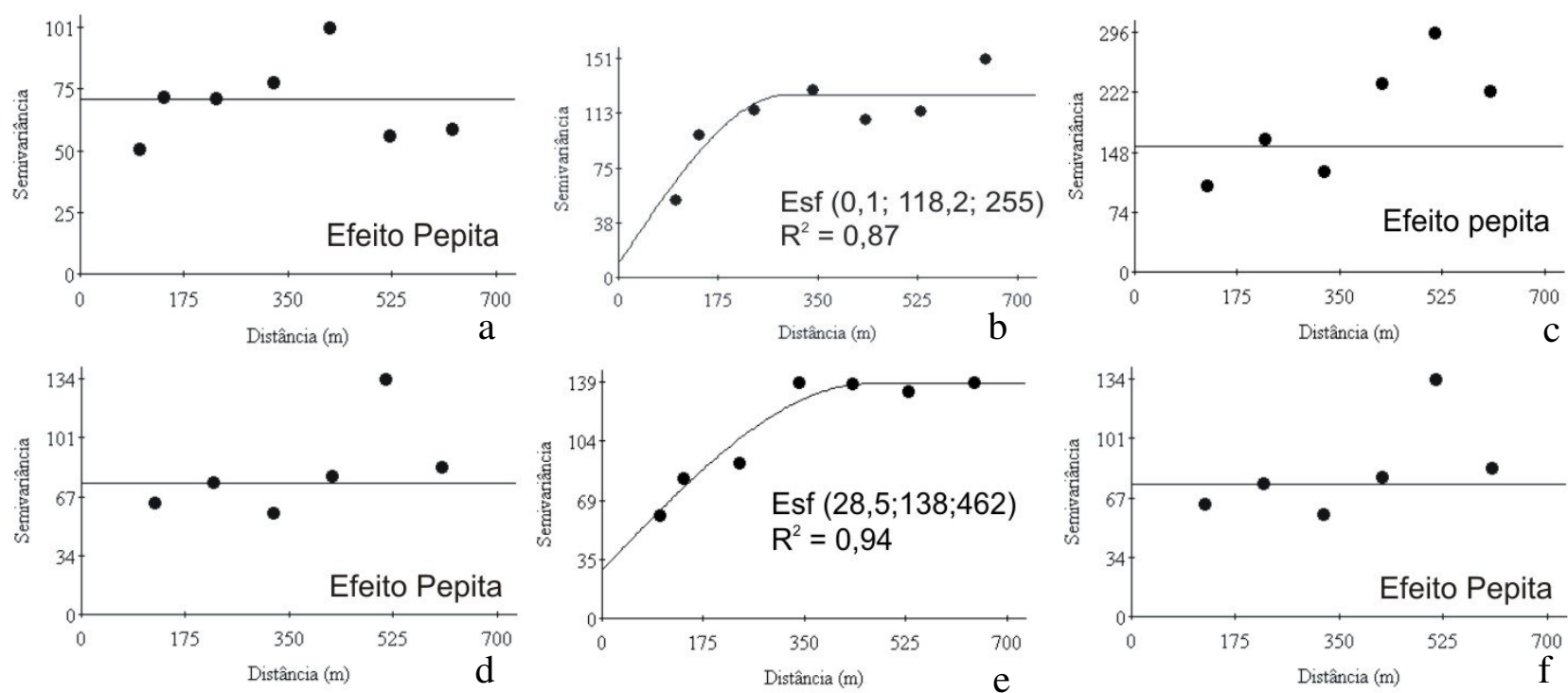

FIGURA 2. Semivariogramas ajustados para os valores de V (\%) na condição de antes (a, c, e) e após (b, d, f) a aplicação em doses variadas, nos talhões A, B e C, respectivamente. Valores entre parênteses são, respectivamente, efeito pepita, patamar e alcance.

O talhão $\mathrm{C}$ apresentou ausência total de dependência espacial (efeito pepita puro), ou seja, distribuição aleatória para os teores de $\mathrm{P}$ e valores de $\mathrm{V}$, antes e depois de realizadas a fosfatagem $\mathrm{e}$ a calagem em doses variadas (Figuras 1 e 2). Esse fato, provavelmente, ocorreu em função da maior variabilidade espacial dos atributos do solo intrínseco dessa área, devido à ocorrência de dois diferentes solos nesse talhão. Isso pode significar que os valores de alcance para os teores de $\mathrm{P}$ e valores de $\mathrm{V}$, no talhão $\mathrm{C}$, foram menores do que o menor espaçamento entre amostras. Esse fato indica que a intensidade de amostragem igual a uma amostra por hectare pode não ter sido suficiente para representar a dependência espacial dos teores de $\mathrm{P}$ e valores de $\mathrm{V}$ no talhão $\mathrm{C}$. $\mathrm{O}$ mesmo fato foi observado para os valores de $\mathrm{V}$ antes e depois de realizada a calagem em doses variadas no talhão $\mathrm{A}$. $\mathrm{O}$ valor do efeito pepita representa a variância não explicada ou ao acaso, freqüentemente causada por variações dos atributos as quais não podem ser detectadas na escala de 
amostragem utilizada ou por erros de medições. Contudo, é impossível quantificar qual contribui mais, se os erros de medição ou a variabilidade em escala menor que aquela amostrada (VIEIRA, 2000).

Conhecido o semivariograma das variáveis e constatada a dependência espacial entre as amostras, foi possível interpolar valores em qualquer posição no campo de estudo por meio da krigagem, e, partindo-se dessas estimativas, foram construídos os mapas de isolinhas para cada uma delas. Quando a variável apresentou ausência de dependência espacial (distribuição aleatória), utilizou-se do método de interpolação linear baseada no inverso do quadrado das distâncias para estimar os valores das variáveis em locais não-amostrados.

Analisando-se os mapas (Figuras 3; 4; 5; 6; 7 e 8), pode-se observar a diferença dos padrões de ocorrência da distribuição espacial entre os teores de $\mathrm{P}$ no solo e os valores de $\mathrm{V}$ para os diferentes talhões. Em algumas situações, esse fato proporciona visualização de diferentes zonas homogêneas de manejo para os atributos analisados, o que permite a adoção do sistema de agricultura de precisão na área do estudo.

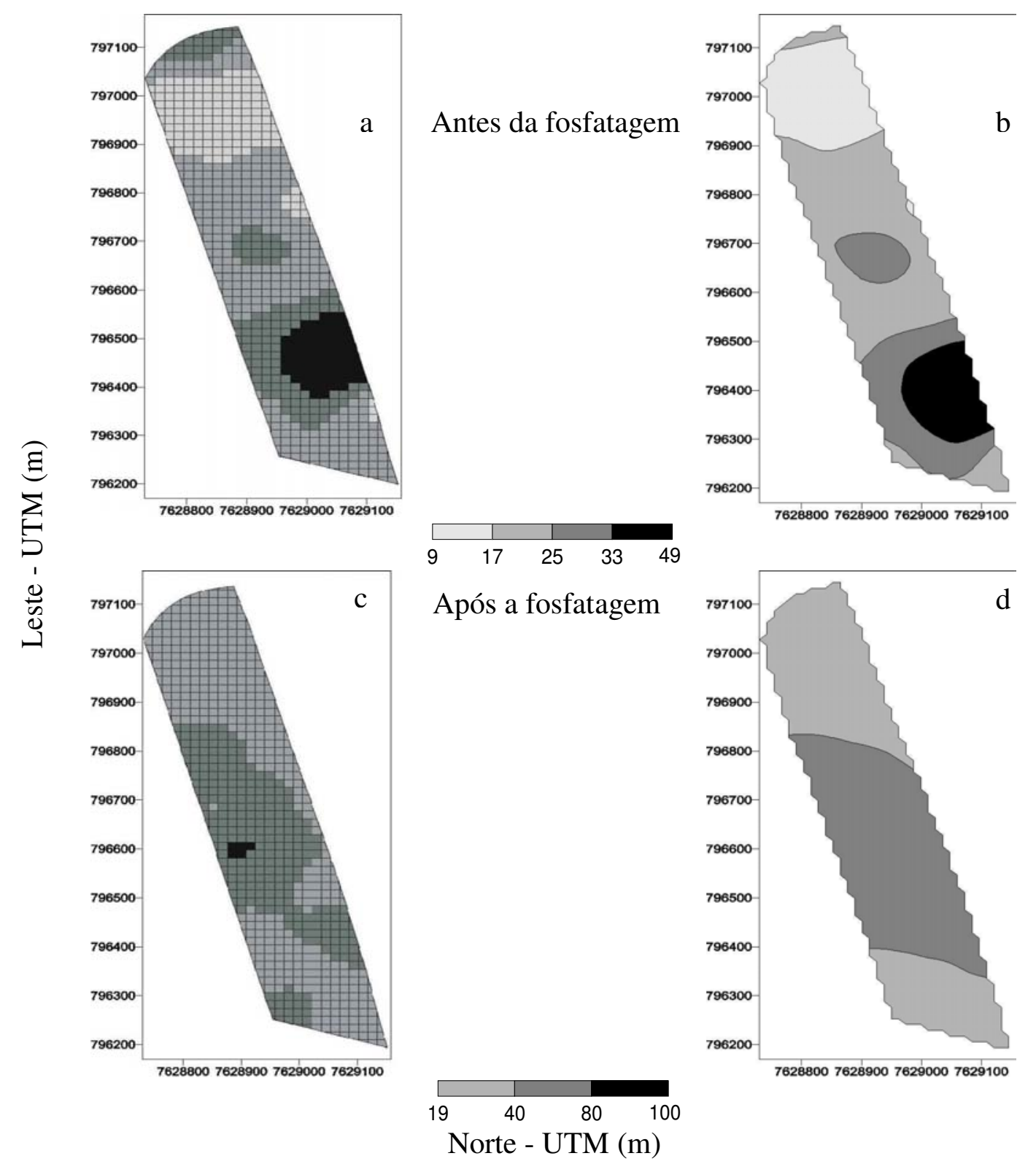

FIGURA 3. Mapas de isolinhas dos valores de $\mathrm{P}\left(\mathrm{mg} \mathrm{dm}^{-3}\right)$, antes e após a fosfatagem em doses variadas, no talhão A. Os mapas a e c foram gerados utilizando-se do programa SSToolbox, e os mapas b e d, utilizando-se dos parâmetros do semivariograma obtidos pela análise geoestatística. 
Observou-se maior continuidade na distribuição espacial dos teores de $\mathrm{P}$ no solo depois de realizada a fosfatagem em doses variadas (manejo regionalizado), comparado aos valores $\mathrm{V}$. Esse fato foi mais evidente nos talhões A e B, acompanhando o aumento dos valores de alcance da dependência espacial dos atributos após o manejo regionalizado. $\mathrm{O}$ talhão $\mathrm{C}$ sempre apresentou ausência total de dependência espacial para esses atributos, comparado aos demais talhões.

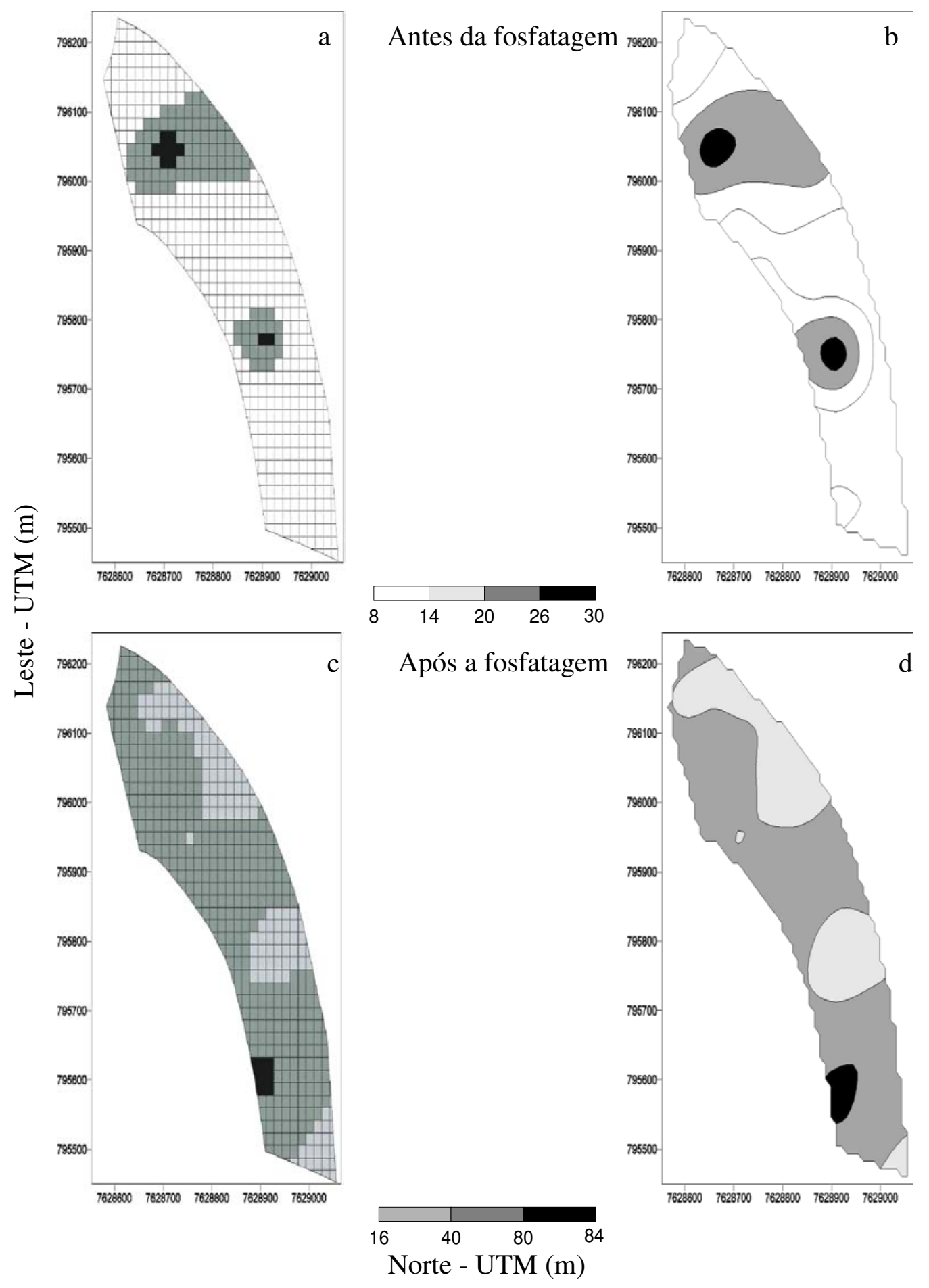

FIGURA 4. Mapas de isolinhas dos valores de $\mathrm{P}\left(\mathrm{mg} \mathrm{dm}^{-3}\right)$, antes e após a fosfatagem em doses variadas, no talhão B. Os mapas a e c foram gerados utilizando-se do programa SSToolbox, e os mapas b e d, utilizando-se dos parâmetros do semivariograma obtidos pela análise geoestatística. 
Os mapas de isolinhas construídos pelo programa SStoolbox, que utiliza interpolador linear para estimar valores em locais não medidos, apresentaram diferentes padrões de ocorrência da distribuição espacial dos atributos, comparados aos demais mapas construídos, levando-se em conta a existência de dependência espacial dos atributos expressa nos semivariogramas. Como era de se esperar, nas situações em que os atributos apresentaram ausência de dependência espacial, os mapas construídos pelo programa SSToolbox foram muito semelhantes aos demais, pois foram construídos utilizando-se do interpolador linear para estimar os valores em locais não-amostrados.

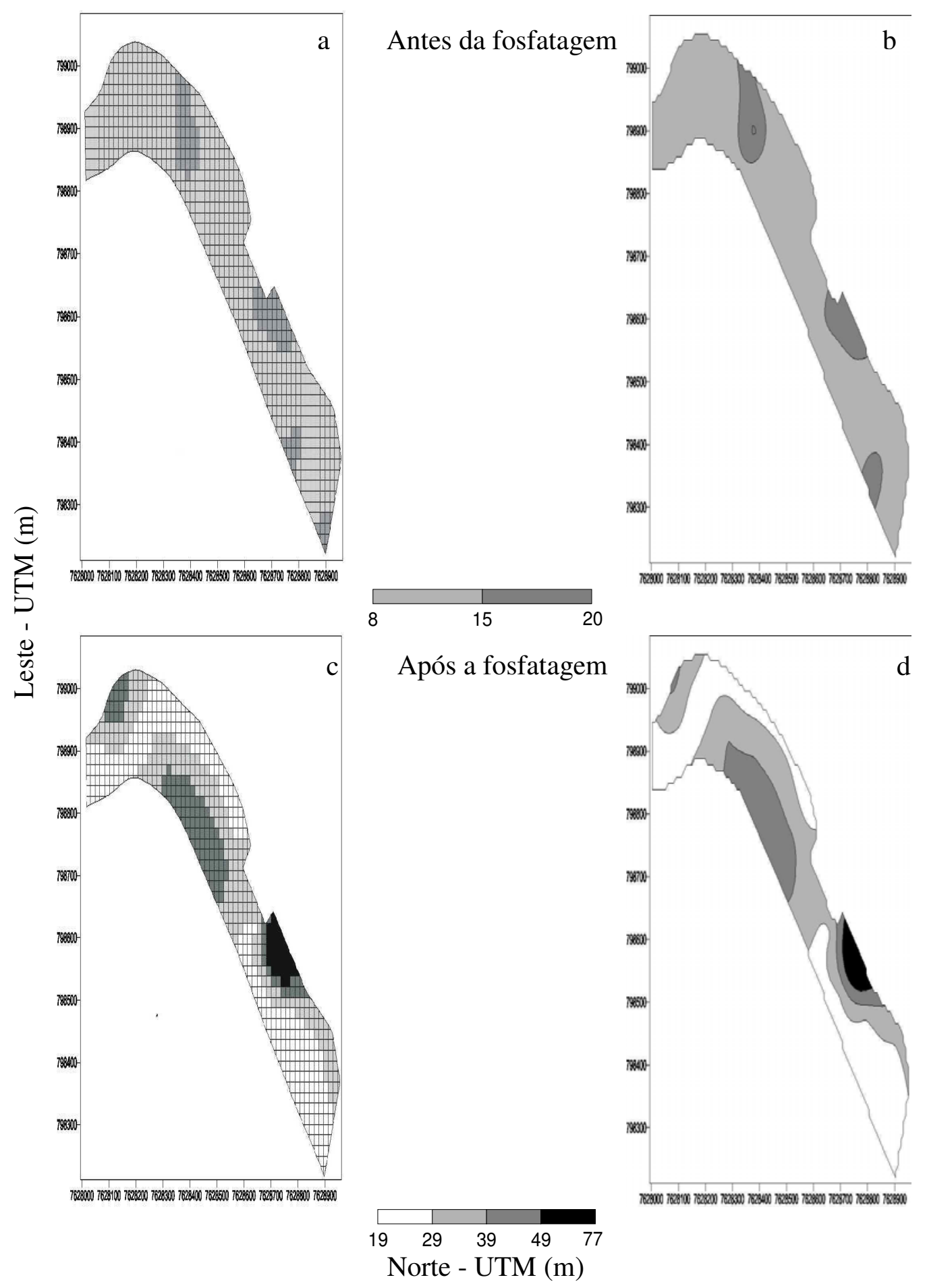

FIGURA 5. Mapas de isolinhas dos valores de $\mathrm{P}\left(\mathrm{mg} \mathrm{dm}^{-3}\right)$, antes e após a fosfatagem em doses variadas, no talhão $\mathrm{C}$. Os mapas a e $\mathrm{c}$ foram gerados utilizando-se do programa SSToolbox, e os mapas b e d, utilizando-se de interpolação linear baseada no inverso do quadrado das distâncias para estimar os valores das variáveis em locais nãoamostrados. 
A precisão dos mapas é dependente do método de interpolação utilizado na estimativa dos valores em locais não-amostrados. O método de interpolação, por sua vez, é dependente da densidade de pontos amostrados por área, isto é, da intensidade da amostragem e, conseqüentemente, da distância entre os pontos amostrados.

No presente estudo, nas situações em que não foi observada dependência espacial entre as amostras, a precisão dos mapas ficou prejudicada, pois acredita-se que o motivo pelo qual foi observado o efeito pepita puro em algumas situações, como no talhão $\mathrm{C}$, foi a baixa intensidade de amostragem utilizada neste estudo, ou seja, uma amostra por hectare, que pode não ter sido suficiente para determinar a dependência espacial dos atributos estudados.

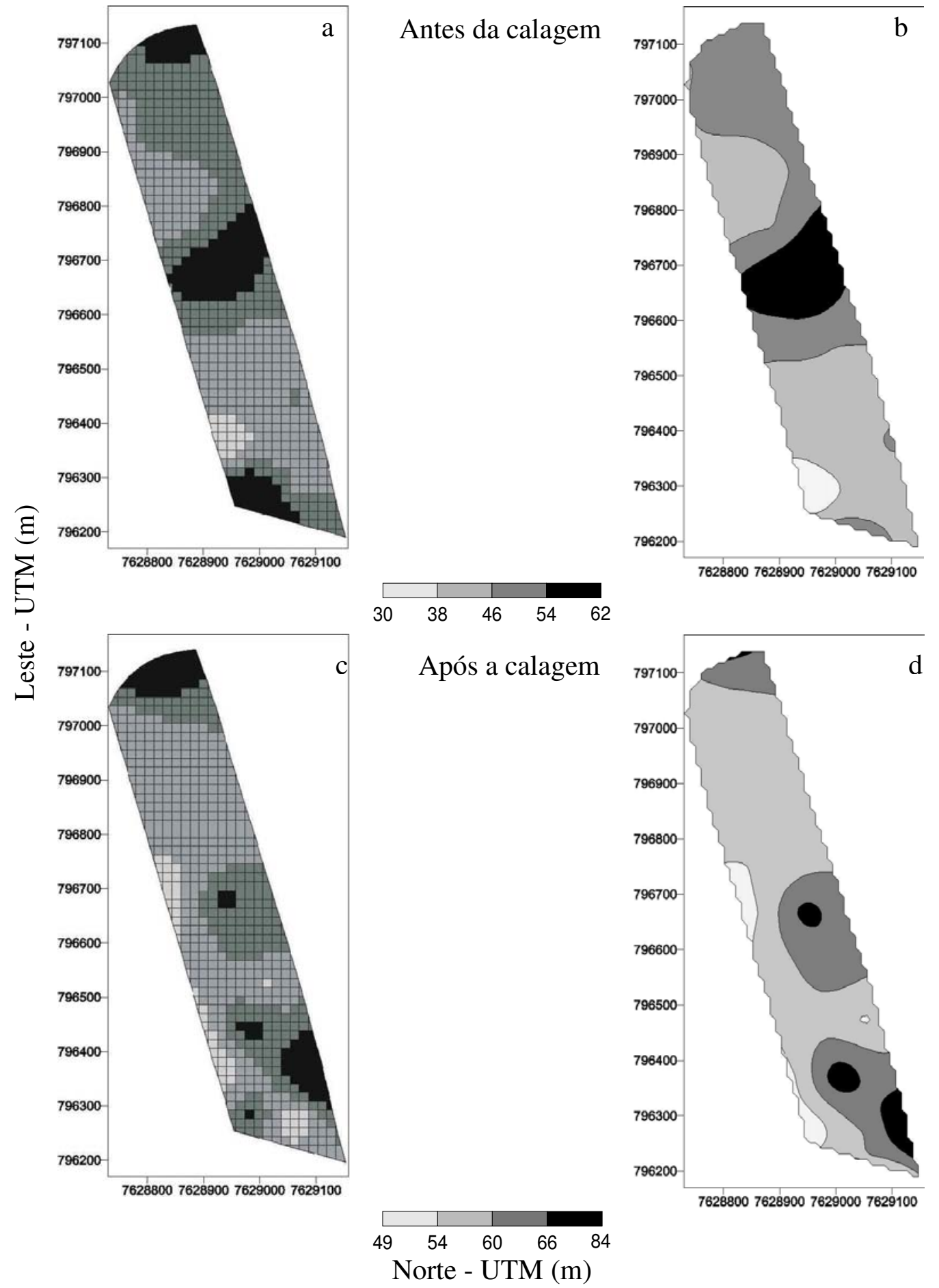

FIGURA 6. Mapas de isolinhas dos valores de V(\%), antes e após a calagem em doses variadas, no talhão A. Os mapas a e c foram gerados utilizando-se do programa SSToolbox, e os mapas b e d, utilizando-se de interpolação linear baseada no inverso do quadrado das distâncias para estimar os valores das variáveis em locais não-amostrados. 
Nesses casos, o método de interpolação baseado em combinação linear, utilizado para estimar os valores em locais não-amostrados, é fonte de imprecisão nos mapas. A forma na qual os dados variam espacialmente no campo, não necessariamente segue equações lineares ou polinômios (VIEIRA, 2000). Pelo contrário, estudos têm mostrado que atributos do solo, geralmente, ocorrem no espaço segundo arranjo estrutural com uma dimensão característica, que é seu domínio, e que corresponde a distância dentro da qual há interdependência dos valores medidos, fato esse que é revelado no semivariograma. Portanto, acredita-se que os mapas construídos por meio da krigagem ordinária, que, levando-se em conta a dependência espacial dos atributos, apresentem maior precisão, pois a krigagem ordinária possibilita a estimativa dos valores em qualquer posição dentro do campo de estudo sem tendência e com variância mínima, proporcionando, dessa maneira, maior precisão na confecção dos mapas.

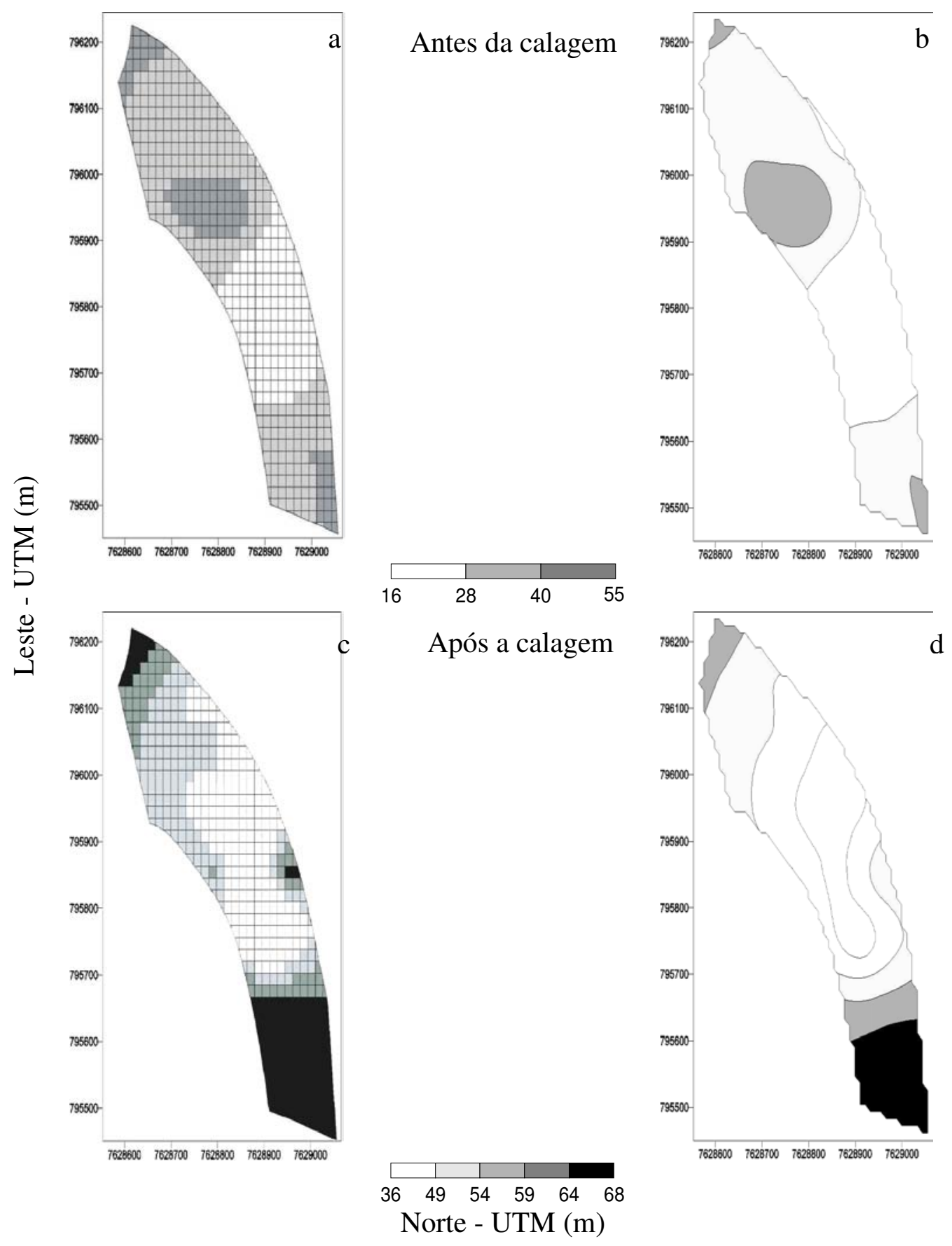

FIGURA 7. Mapas de isolinhas dos valores de V (\%), antes e após a calagem em doses variadas, no talhão B. Os mapas a e c foram gerados utilizando-se do programa SSToolbox, e os mapas b e d, utilizando-se dos parâmetros do semivariograma obtidos pela análise geoestatística. 

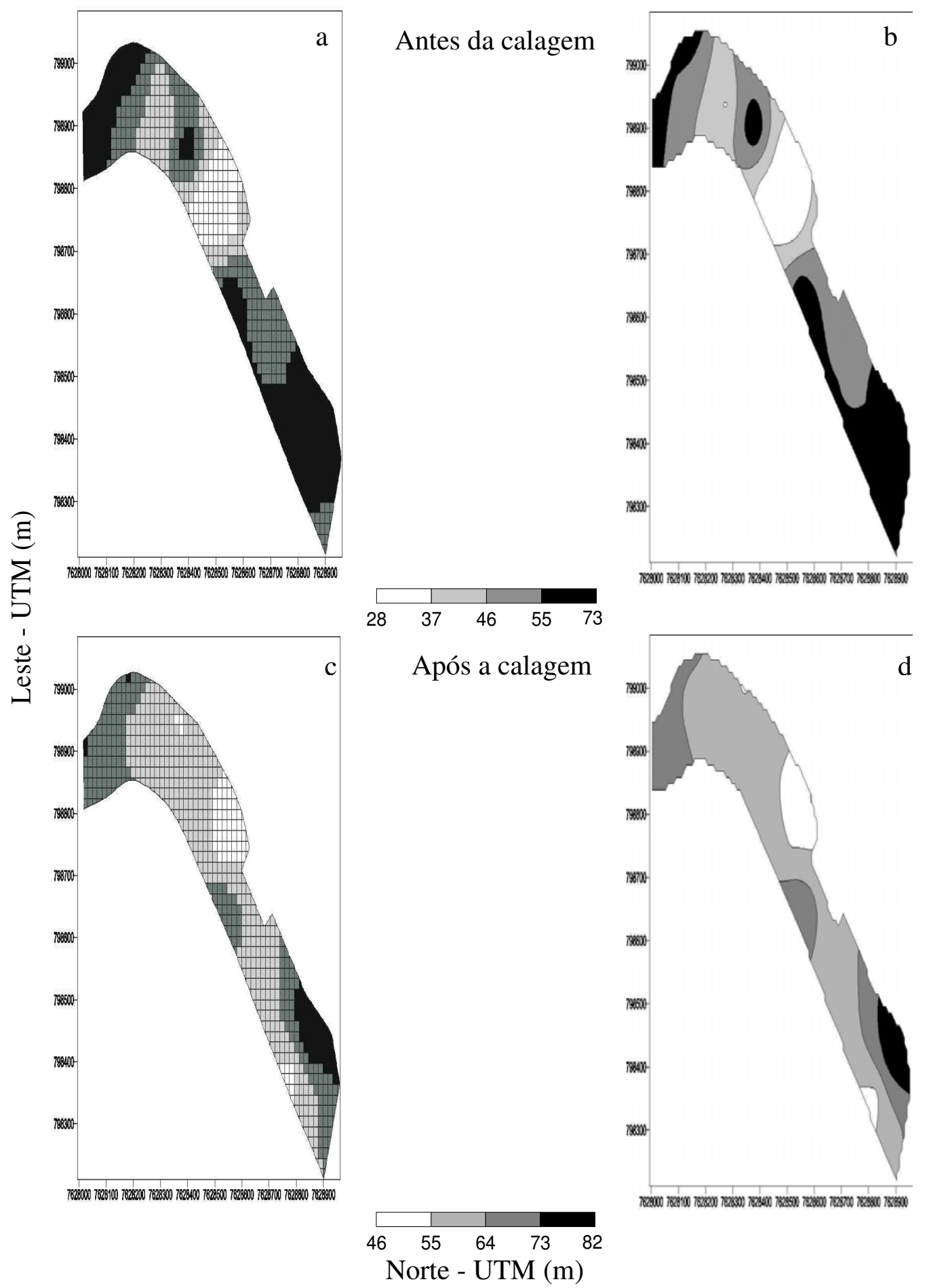

FIGURA 8. Mapas de isolinhas dos valores de V(\%), antes e após a calagem em doses variadas, no talhão C. Os mapas a e c foram gerados utilizando-se do programa SSToolbox, e os mapas b e d, utilizando-se da interpolação linear baseada no inverso do quadrado das distâncias para estimar os valores das variáveis em locais não-amostrados.

\section{CONCLUSÕES}

Os mapas de isolinhas elaborados por meio da krigagem, levando-se em consideração a existência de dependência espacial de atributos do solo, expressa no semivariograma, apresentaram maior precisão e diferentes padrões de ocorrência da distribuição espacial dos atributos, comparados aos mapas de isolinhas construídos utilizando-se do interpolador linear para estimativa dos valores em locais não medidos. 
A densidade de amostragem igual a uma amostra por hectare não foi suficiente para revelar a dependência espacial dos teores de $\mathrm{P}$ e valores de $\mathrm{V}$ no talhão $\mathrm{C}$, assim como para os valores de $\mathrm{V}$ no talhão A.

Os talhões A e B apresentaram maior continuidade espacial para os teores de $\mathrm{P}$ no solo, e o talhão B, maior continuidade espacial para os valores de V.

Houve aumento da continuidade espacial dos teores de $\mathrm{P}$ no solo e valores de $\mathrm{V}$ depois de realizadas a calagem e a fosfatagem em doses variadas.

\section{AGRADECIMENTOS}

Os autores agradecem à Coordenação de Aperfeiçoamento de Pessoal de Nível Superior (CAPES), pela bolsa de estudo do primeiro autor, e à Fazenda Santa Isabel, na pessoa do Eng ${ }^{\circ}$ Agrônomo Paulo de Araújo Rodrigues, pela concessão da área experimental e total apoio nos trabalhos de campo.

\section{REFERÊNCIAS}

BURGESS, T.M.; WEBSTER, R. Optimal interpolation and isarithmic mapping of soil properties I - The semivariogram and punctual kriging. Journal of Soil Science, Madison, v.31, n.2, p.315-31, 1980.

CAMBARDELLA, C.A.; MOORMAN, T.B.; NOVAK, J.M.; PARKIN, T.B.; KARLEN, D.L.; TURCO, R.F.; KONOPKA, A.E. Field-scale variability of soil properties in central Iowa soils. Soil Science Society American Journal, Madison, v.58, n.5, p.1501-11, 1994.

CRESSIE, N. Statistics for spatial data. New York: John Wiley, 1991. 900 p.

GOLDEN SOFTWARE. Surfer for Windows: Surface mapping system. Versão 6.01. Golden, 1995. Não paginado.

NATALE, W.; COUTINHO, E.L.M. Avaliação da eficiência agronômica de frações granulométricas de um calcário dolomítico. Revista Brasileira de Ciência do Solo, Campinas, v.18, n.1, p.55-62, 1994.

PRADO, H. Levantamento detalhado de solos da Fazenda Santa Isabel. Campinas: CATI, 1994. $3 \mathrm{v}$.

ROBERTSON, G.P. $G S^{+}$: Geostatistics for the environmental sciences. Versão 5.03 beta. Plainwell: Gamma Design Software, 1998. 152 p.

SHAPIRO, S.S.; WILK, M.B. An analysis of variance test for normality: complete samples, Biometrika, London, v.52, n.3-4, p.591-611, 1965.

SCHLOEDER, C.A.; ZIMMERMAN, N.E.; JACOBS, M.J. Comparison of methods for interpolating soil properties using limited data. Soil Science Society American Journal, Madison, v.65, n.2, p.470-9, 2001.

SOUZA, L.S.; COGO, N.P.; VIEIRA, S.R. Variabilidade de fósforo, potássio e matéria orgânica no solo em relação a sistemas de manejo. Revista Brasileira de Ciência do Solo, Campinas, v.22, n.1, p.77-86, 1998.

TRANGMAR, B.B.; YOST, R.S.; UEHARA, G. Application of geostatistics to spatial estudies of soil properties. Advances in Agronomy, San Diego, v.38, n.1, p.45-93, 1985.

VIEIRA, S.R. Geoestatística em estudos de variabilidade espacial do solo. In: NOVAIS, R.F.; ALVAREZ, V.V.H.; SCHAEFER, C.E.G.R. Tópicos em ciência do solo. Viçosa: Sociedade Brasileira de Ciência do Solo, 2000. p.1-54. 